Ann. Zootech., I978, 27 (I), Io7-II3.

\title{
Note
}

\section{Enregistrement des activités alimentaires et méryciques des ovins au pâturage}

\author{
G. BÉCHET \\ avec la collaboration technique de J. Fi,icher et E. Drez \\ Station de Recherches sur l'Élevage des Ruminants, \\ Centre de Recherches de Clermont-Ferrand, I.N.R.A. \\ Theix, 63110 Beaumont (France)
}

\section{Résumé}

L'étude des activités alimentaires et méryciques des ovins au pâturage a été facilitée par l'utilisation d'un vibrographe modifié pour enregistrer les mouvements de la mâchoire inférieure. Cet appareil de faible poids est fixé sur le dos de l'animal à l'aide d'un harnais et d'une besace de toile. Il a une autonomie de 24 heures et l'enregistrement s'effectue sur un disque de papier.

Les mouvements de la mâchoire sont transmis à un circuit d'air composé d'une poire de caoutchouc maintenue à la mâchoire à l'aide d'un licol, et reliée par un tuyau à un système cylindre-piston incorporé au vibrographe. Tous les éléments de ce circuit sont en matière plastique, nylon ou latex, présentant une bonne étanchéité et une grande résistance aux chocs et perturbations de toutes sortes.

\section{Introduction}

L'étude des activités alimentaires et méryciques des ruminants au pâturage a été facilitée par l'enregistrement à 1'aide d'appareils autonomes soit des mouvements de la tête de l'animal (Alrdoex, I962; O'SheA, i968; Stobbs, I970; $Z$ EeB et Z IMMERLANN-MIULrER, I97I), soit des mouvements de la mâchoire inférieure (Ruckebusch et Bueso, I973; Favre, I974).

Cette dernière méthode est la seule qui permette d'obtenir des informations précises sur l'ingestion et la rumination au pâturage. Elle a été utilisée sur les 
bovins par RUCKEBUSCH et BUENo (I973). Ceux-ci ont placé dans un vibrographe (enregistreur de vibrations) un système "cylindre-piston " constitué d'une seringue de verre, afin que l'appareil enregistre les variations de pression d'air venant d'un ballonnet situé en position sous-mandibulaire sur l'alliance d'un licol.

Nous avons adapté ce dispositif aux ovins en réduisant la fragilité du système par l'emploi d'éléments uniquement en matière plastique, et en améliorant son. étanchéité et sa sensibilité.

\section{Matériel d'enregistrement}

Le vibrographe KIENZLF (I) TFW 3/8, décrit par RUCKebUSch et BUENo (I973) a la forme d'un boîtier de $14,4 \mathrm{~cm}$ de diamètre pesant $960 \mathrm{~g}$; il est constitué de deux parties :

- l'une comportant une horloge qui transmet un mouvement uniforme à un pignon de sortie sur lequel on fixe quotidiennement un disque de papier;

- l'autre constituée d'un balancier muni d'un ressort de rappel et d'un stylet inscripteur, l'ensemble se déplaçant sur un pignon de guidage.

Le mouvement d'horlogerie entraîne le pignon de guidage et fait décrire au stylet, sur le disque de papier, une spirale à 8 tours en 24 heures formant la ligne de base. Le stylet inscrit les oscillations du balancier sous la forme d'un trait de $0,2 \mathrm{~cm}$, perpendiculaire à la ligne de base.

Nous avons transformé l'appareil pour recevoir les mouvements de la mâchoire par transmission pneumatique : un circuit d'air fermé constitué d'une poire de caoutchouc fixée sur un licol en position sous-mandibulaire reliée par un tuyau de matière plastique à un piston disposé à l'intérieur du vibrographe. Les modifications apportées au vibrographe sont décrites dans le tableau suivant, complété par la figure $I$ et le schéma $I$.

\begin{tabular}{l|l}
\hline $\begin{array}{l}\text { Partie du boîtier compre- } \\
\text { nant le balancier. }\end{array}$ & $\begin{array}{l}\text { Percée d'un orifice de IO-I50 SI (2) dans lequel est introduit unt } \\
\text { boulon de nylon transpercé longitudinalement et qui constitue } \\
\text { la base d'un système cylindre-piston. }\end{array}$ \\
\hline Balancier. & Allégé. \\
\hline Ressort de rappel. & Déplacé et allongé. \\
\hline
\end{tabular}

L'extrémité du boulon de nylon située à l'extérieur de l'appareil est décolletée ( $\varnothing 0,80 \mathrm{~cm}$ ) et reçoit un tuyau de PVC (3) souple, l'autre extrémité cylindrique $(\varnothing \mathrm{I}, 43 \mathrm{~cm}$ ) forme une buse d'entrée de l'appareil. Elle est recouverte d'un doigt de gant chirurgical en latex de taille 6 , et introduite en force dans un cylindre de matière plastique constitué par le corps d'une seringue de ro ml. Le piston de la seringue est renversé, le joint de caoutchouc à son extrémité est poli en forme de

(1) Commercialisé en France par la Socicté d'appareils Fietzle France. \%one industrielle, 8 , avenue Gay-I,ussac, 9I380 Chilly Mazarin, Télex 690423.

(2) SI : Système international.

( ${ }^{3}$ ) Chlorure de polyvinyl. 
cône et réduit de diamètre, rendant ainsi le piston plus mobile. Il vient s'appliquer contre le doigt de gant.

En se remplissant d'air, le doigt de gant repousse le piston qui imprime un mouvement au balancier.

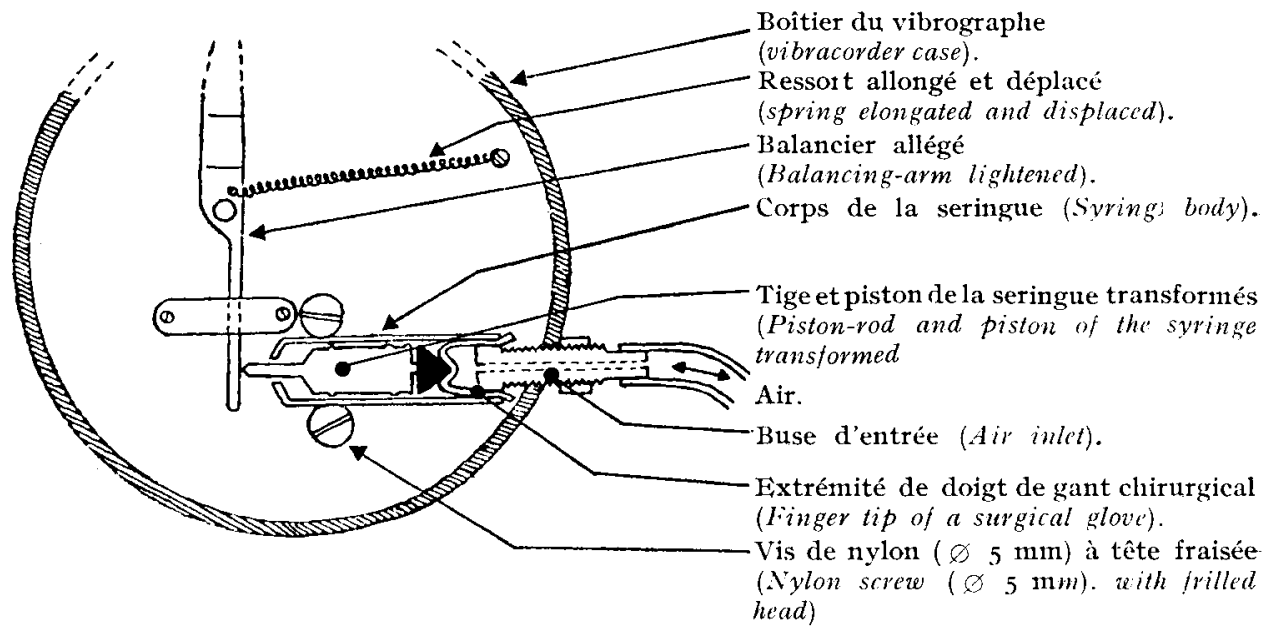

Schlima I. - Modifications apportées au vibrographe.

Modifications to the vibracorder.

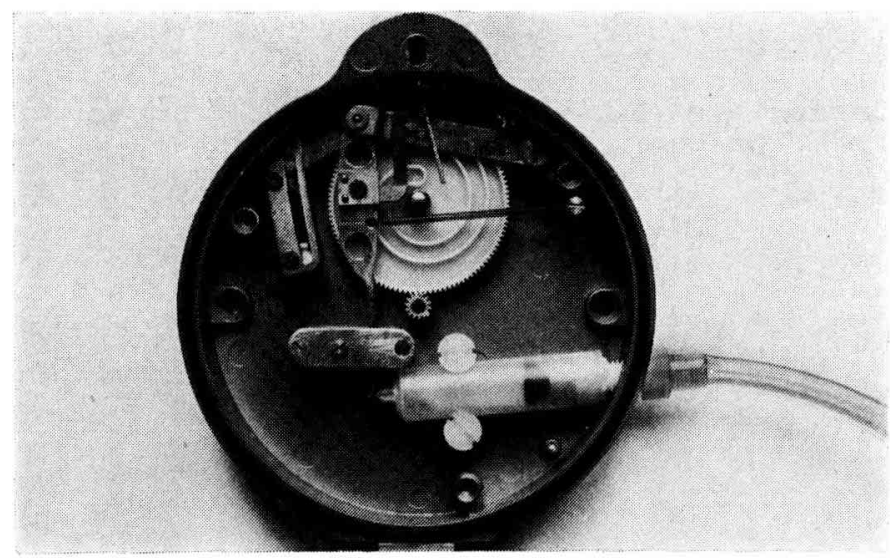

FIG. I. - Partie modifiée du vibrographe.

The modified part of the vibracorder.

Cette réalisation en matière plastique est résistante aux chocs. Le latex assure une étanchéité au système et ne craint pas l'humidité; il est très sensible aux variations de pression d'air.

La poire de caoutchouc, transmettant les mouvements de mâchoire, est une poche pour l'appareil d'Orsat (I) de $8 \mathrm{~cm}$ de largeur sur $\mathrm{r} 2 \mathrm{~cm}$ de longueur, remplie

(1) Fabriquée par les É,tablissements Verneret, 31, rue Pierre-et-Marie-Curic, 9420I Ivry. 
de mousse de polyuréthane lui donnant une forme arrondie. Elle est munie à sa sortie d'un tube semi-rigide de polyéthylène $(\infty 0,4 \times 0,8 \mathrm{~cm})$ sur lequel est fixé un tuyau de PVC souple de $\varnothing 0,5 \times I \mathrm{~cm}$ et de $80 \mathrm{~cm}$ de longueur la reliant à la buse d'entrée du vibrographe.

\section{Fixation du dispositif et utilisation sur les brebis au pâturage}

Le licol est composé de lanières de nylon, ne s'allongeant pas, comme le cuir, sous l'effet de la pluie, et reliées à une base rigide en PVC en robée de toile (schéma 2 ). La poire de caoutchouc est fixée sur cette base à l'aide de plastobande (toile encollée).
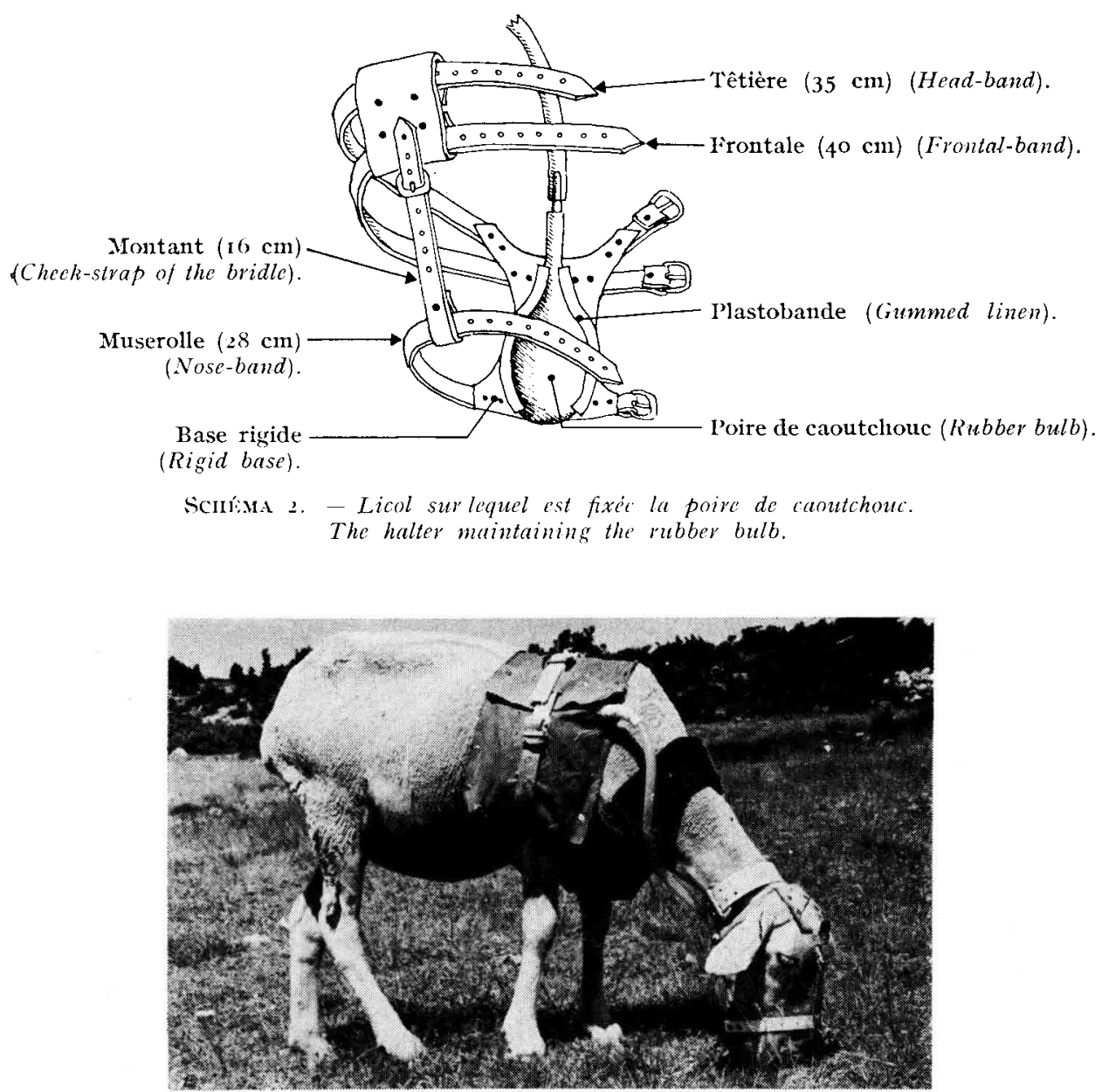

Fig. 2. - Brebis au pâtutage équipéc pour l'envegistrement. Grazing animal équiped for recording. 
I,e vibrographe, isolé de l'humiclité dans un sac de polyvinyl est enfermé dans la poche d'une besace de toile qui est fixée sur le dos de la brebis par un harnais analogue à ceux utilisés pour les sacs à fèces ('T'Issier, Béchét, Molénat, I975). I, autre poche de la besace contient un contre-poids (sable...) on un appareil pour d'autres mesures (fig. 2).

Ainsi équipées, les brebis peuvent pâturer en toute liberté et le dispositif présente une botnne résistance aux secousses, à la plutie ou à d'autres perturbations. Ij'emploi du temps s'inscrit sur le clisque de papier qui est clangé sans difficulté chaque jour.

Les enregistremonts obtenuss sur les discques du vibrographe de type 'lFW $3 / 8$ montrent aisément les activités d'ingestion, de rumination ou le repos (fig. 3). Ceci était plus difficile sur les en registrements effectués auparavant avec des appareils de type $2.4 / 8$ d'unc autonomic de 8 jours, mais tournant $\&$ fois moins vite.

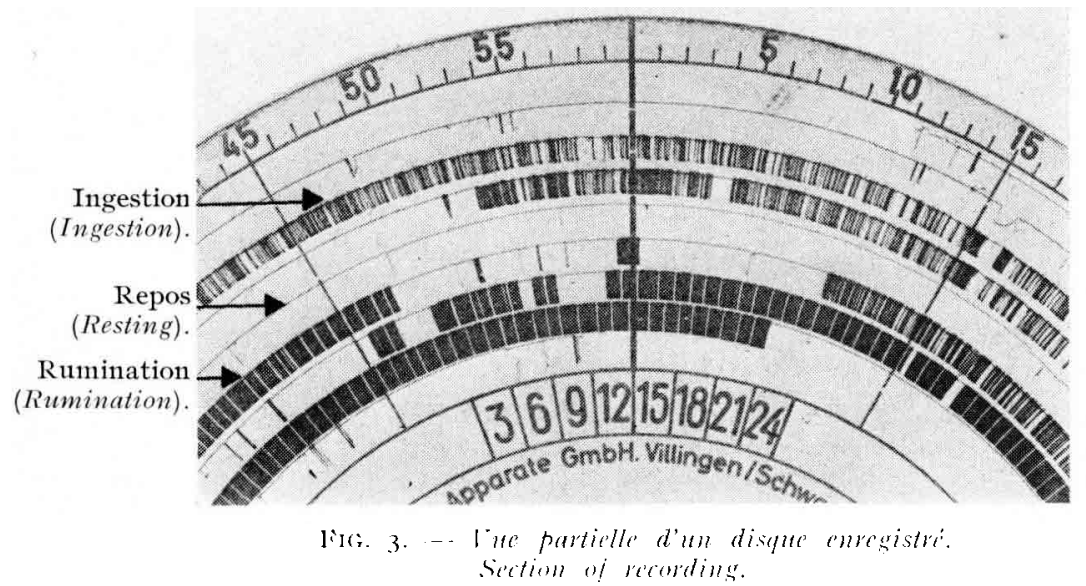

Un lecteur de disque permet également, à l'aicle d'un curseur, de totaliser rapidement les temps journaliers d'ingestion, de rumination ou de repos.

les mesures ont ainsi été effectuées sur les parcours du Causse du Irarzac pendant les deux dernières années. Nous présentons ici, à titre d'exemple, quel-

TAII,TIST I

Paramitres de l'ativiti alimantaire

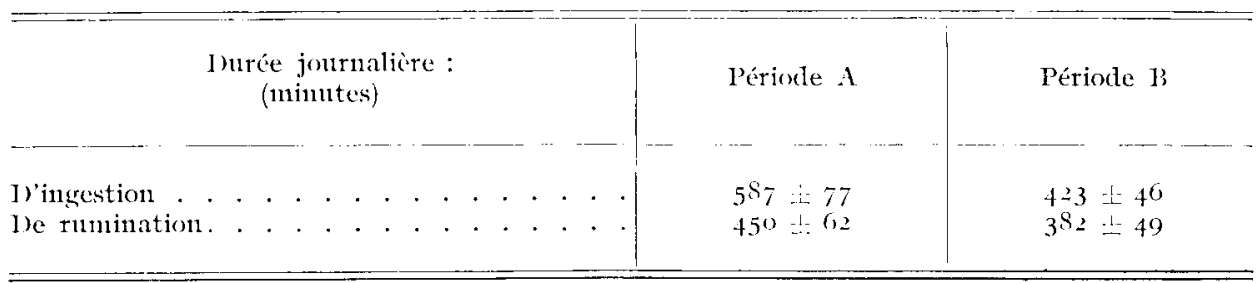


ques résultats obtenus au cours de deux périodes d'une semaine chacunc (tabl. I, schéma 3) :

- période A : mesures effectuées fin mai sur un troupeau de brebis allaitantes; taries.

- période B : mesures effectuées début aont sur le même troupeau de brebis

Le temps a été beau durant ces deux périodes et les conditions d'éclairement. lunaire ont été sensiblement les mêmes.
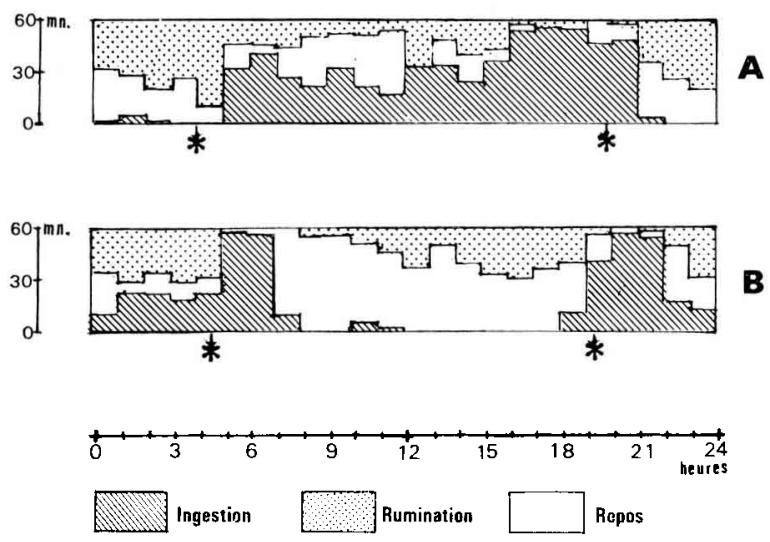

* : lever et coucher du soleil.

Période A : fin mai : brebis allaitantes (Fnd of May recording: suckling takes).

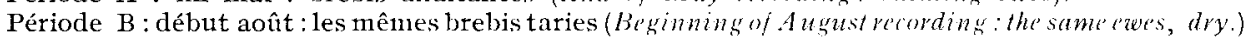

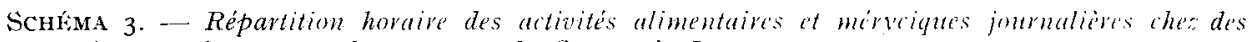
ovins au pâturage sur les parcours du Causse du Laraat.

Hourly variation of the daily feeding activity and rmmination of grasing shesp on the' Catuse du Larzac pastures.

Cet appareil, avec une seule intervention journalière permettra d'obtenir des données sur les activités alimentaires et méryciques au pâturage, ce qui était limité jusqu'ici par le problème de la présence humaine en permanence sur le terrain d'expérience.

Accepté pour publication en Noumber 1977.

\section{Summary}

Recording of feeding activity and rumination in grazing sheep.

Feeding activity and rumination of grazing sheep was studied by a vibracorder modified for recording the lower jaw movements. This light apparatus was placed in a canvas bag on the back of the animals and held in place with a harness (fig. I and 2).

The jaw movements were transmitted via an air circuit composect of a rubber bulb maintained on the jaw with a halter, and connected by a tube to a cylinder piston system in the vibracorder (diagrams I and 2). All these circuit components are in plastic, nylon and latex and thus highly watertight and in addition high shock resistant.

The recorder paper was changed every day and the feeding activity and rumination easily discerned (fig. 3). 


\section{Références bibliographiques}

AlLDFN W. G., r962. Rate of herbage intake and grazing time in relation to herbage availability. Proc. Aust. Soc. Anim. Prod., 4, I63-166.

FAVRE Y., I974. Comportement des bovins en alpage. in HENRY J. M. Inst. Nat. d'études rurales montagnardes. C.T.G.R.E.F., Grenoble.

O'ShEA J., I968. A simple timing device for use in behavioural 'studies with grazing animals. Vet. Rec., 83, I 54-I 55 .

RUCKEBUSH Y., BUENo L., 1973. Un dispositif simple et autonome d'enregistrement de l'activité alimentaire chez les bovins au pâturage. Ann. Rech. Vet., 4, 627-636.

SToвbs T. H., I970. Automatic measurement of grazing time by dairy cows on tropical grass and legume pastures. Tropical Grasslands, 4(3), 237-244.

Tisster M., BÉchet G., Molénat G., 1975. Appareils de collecte totale de fèces pour agneaux en allaitement ou à l'engrais et pour brebis. Ann. Zootech., 24, 595-602.

ZEEB K. et ZIMMERLANy-MCLLER M. (5 mai r97I). La structure sociale et l'activité des vaches laitières. Der Tievzüchter, $\mathrm{n}^{\mathrm{o}} \mathrm{g}$. 